\title{
A THEOREM OF ALIEV
}

A. C. PETERSON

1. Introduction. We are concerned with the $n$th $(n \geqq 3)$ order linear differential equation

$$
l_{n}[y] \equiv y^{(n)}+\sum_{k=0}^{n-1} p_{k}(x) y^{(k)}=0
$$

where the coefficients are continuous on $(-\infty, \infty)$. The results in this paper generalize the well-known result that the first conjugate point $\eta_{1}(t)$ for $l_{3}[y]=0$ satisfies

$$
\eta_{1}(t)=\min \left[r_{21}(t), r_{12}(t)\right]
$$

(see Definition 2). Aliev [1] proved for $l_{4}[y]=0$ that

$$
r_{1111}(t)=\min \left[r_{121}(t), r_{112}(t)\right]
$$

and purported to prove that

$$
r_{1111}(t)=\min \left[r_{211}(t), r_{112}(t)\right]
$$

but his proof is incorrect. Since $\eta_{1}(t)=r_{1111}(t)$ [2], [3], we have $\eta_{1}(t)$ $=\min \left[r_{121}(t), r_{112}(t)\right]$. Theorem 1 gives a much easier proof of this result, establishes the validity of (1) (this was left as an open question in [4]), and gives an $n$th order generalization of these results. The simplicity of the Theorem 1 is due to a theorem of Sherman [5] which gives that if $b>\eta_{1}(t)$, then there is a nontrivial solution of $l_{n}[y]=0$ with a simple zero at $t$ and whose first $n$ zeros on $[t, b)$ are simple zeros.

2. Definitions and main result. Before we state the main result we make the following definitions.

Definition 1. A nontrivial solution $y$ of $l_{n}[y]=0$ is said to have a $i_{1}-i_{2}-\cdots-i_{\nu}\left(\nu=2, \cdots, n, \sum_{k=1}^{\nu} i_{k}=n, 1 \leqq i_{\nu} \leqq n-1\right)$ distribution of zeros on $[t, b]$ provided there are numbers $t_{1}, \cdots, t_{\nu}$ such that $t \leqq t_{0}<t_{1}<\cdots<t_{\nu} \leqq b$ and $y$ has a zero at each $t_{k}$ of order at least $i_{k}$.

Definition 2. The extended real number $r_{i_{1} i_{2} \cdots i_{\nu}}(t)$ is the infimum of the set of $b>t$ such that there is a nontrivial solution $y$ of $l_{n}[y]=0$ having an $i_{1}-i_{2}-\cdots-i_{\nu}$ distribution of zeros on $[t, b]$.

REMARK 1. If $t \leqq t_{1}<t_{2}<\cdots<t_{\nu}<r_{i_{1} i_{2} \cdots i_{\nu}}(t) \leqq \infty$, then there is a unique solution $u(x)$ of $l_{n}[y]=0$ satisfying

$$
y^{(j)}\left(t_{k}\right)=A_{j k}
$$

Presented to the Society, April 26, 1969; received by the editors February 3, 1969. 
$k=1,2, \cdots, \nu ; j=0,1, \cdots, i_{k}$ where the $A_{j k}$ are constants.

DEFINITION 3 . For $1 \leqq p \leqq n-1$

$$
s_{p}(t)=r_{i_{1} i_{2}} \cdots i_{n-1}(t)
$$

where $i_{p}=2$ and $i_{k}=1$ for $k \neq p$.

We now state our main result.

THEOREM 1. For $1 \leqq j \neq k \leqq n-1$

$$
\eta_{1}(t)=\min \left[s_{j}(t), s_{k}(t)\right] .
$$

Proof. If $\eta_{1}(t)=\infty$, then the theorem is obvious and, hence, we can assume that $\eta_{1}(t)$ exists. Clearly $\eta_{1}(t) \leqq \rho(t) \equiv \min \left[s_{j}(t), s_{k}(t)\right]$, and so it suffices to show that the assumption $\eta_{1}(t)<\rho(t)$ leads to a contradiction. By Theorem 1 in [5] there is a nontrivial solution $u(x)$ of $l_{n}[y]=0$ whose first $n$ zeros, say $x_{k}, 1 \leqq k \leqq n$, are simple zeros where $t=x_{1}<x_{2}$ $<\cdots<x_{n}<\rho(t)$. We can assume that $j<k$. Since $t=x_{1}<x_{2}<\cdots$ $<x_{n}<s_{j}(t)$ there is a unique solution $v(x)$ of $l_{n}[y]=0$ satisfying

$$
y\left(x_{i}\right)=0, \quad y^{\prime}\left(x_{j}\right)=0, \quad y\left(x_{k}\right)=1,
$$

where $i=1, \cdots, k-1, k+2, \cdots, n$ unless $k=n-1$ in which case $i=1,2, \cdots, n-2$ (see Remark 1). It is easy to see that $v(x)>0$ for $x_{k} \leqq x \leqq x_{k+1}$. It follows from Lemma 1.1 in [6] that there is a nontrivial linear combination of $u(x)$ and $v(x)$ with a double zero in $\left(x_{k}, x_{k+1}\right)$. But this same linear combination has zeros at $x_{i}$, $i=1, \cdots, k-1, k+2, \cdots, n$ unless $k=n-1$ in which case $i=1,2, \cdots, n-2$. This contradicts $t=x_{1}<x_{2}<\cdots<x_{n}<s_{k}(t)$ and the theorem is proved.

It follows from Theorem 1 that at most one of the numbers $s_{p}(t)$, $1 \leqq p \leqq n-1$, is greater than $\eta_{1}(t)$. Many examples can be given to show that we do not have $\eta_{1}(t)=s_{p}(t)$ for $1 \leqq p \leqq n-1$. For $n=3$ see Hanan [7]. A simple example for $n=4$ is $y^{i v}+y^{\prime}=0$ for which we have $\eta_{1}(t)=s_{1}(t)=s_{2}(t) \approx t+5.9<s_{3}(t)=\infty \quad$ [8]. For those equations of the form

$$
y^{i v}+p(x) y=0, \quad p(x)<0
$$

for which $\eta_{1}(t)$ exists we have $\eta_{1}(t)=s_{1}(t)=s_{3}(t)<s_{2}(t)=\infty$ [4], [6].

\section{REFERENCES}

1. R. G. Aliev, On certain properties of solutions of ordinary differential equations of fourth order, Sb. Aspirantsh, Kazan Institute (1964), 15-30. (Russian).

2. P. Hartman, Unrestricted n-parameter families, Rend. Circ. Mat. Palermo (2) 7 (1958), 123-142. 
3. Z. Opial, On a theorem of O. Arama, J. Differential Equations 3 (1967), 88-91.

4. A. C. Peterson, Distribution of zeros of solutions of a fourth order differential equation, Pacific J. Math. (to appear).

5. T. L. Sherman, Conjugate points and simple zeros for ordinary linear differential equations, Trans. Amer. Math. Soc. (to appear).

6. W. Leighton and $Z$. Nehari, On the oscillation of solutions of self-adjoint linear differential equations of the fourth order, Trans. Amer. Math. Soc. 89 (1958), 325-377.

7. M. Hanan, Oscillation criteria for third-order linear differential equations, Pacific J. Math. 11 (1961), 919-944.

8. R. G. Aliev, On the sign of the Green's function for a boundary-value problem for a fourth order differential equation, Izv. Vysč, Učebn. Zaved. Matematika 1964, no. 6 (43), 3-9. (Russian)

UNIVERSITY OF NEBRASKA 